\title{
Asociación de los niveles de adiponectina y del factor de necrosis tumoral-alfa (TNF-a) con la albuminuria en pacientes con diabetes mellitus tipo 2
}

Association of adiponectin and tumor necrosis factor-alpha (TNF-a)

levels with albuminuria in patients with type 2 diabetes mellitus

\section{Jhoalmis Sierra-Castrillo MSc ${ }^{1}$,} Lyz J. Gómez-Rave MSc ${ }^{2}$

\begin{abstract}
Introducción: la diabetes mellitus tipo 2 es una enfermedad metabólica crónica que de no ser controlada puede generar progresivamente daño renal hasta causar nefropatía diabética. Además, está altamente asociada con un estado inflamatorio permanente relacionado con la secreción de adipocitoquinas (IL-6, TNF- $\alpha$, leptina, adiponectina) que participan en la homeostasis del metabolismo y son determinantes en la regulación del proceso aterogénico y la resistencia a la insulina. Objetivo: relacionar el comportamiento de los niveles de adiponectina y TNF- $\alpha$ con la albuminuria en pacientes con diabetes mellitus tipo 2. Materiales y métodos: la investigación se basó en un modelo descriptivo de corte transversal con una población de estudio conformada por pacientes que ingresaron al Hospital Universitario Erasmo Meoz (Cúcuta, Colombia). De acuerdo con los criterios de selección establecidos se conformaron dos grupos de análisis: pacientes con diagnóstico de diabetes mellitus tipo 2 y albuminuria $>30 \mathrm{mg} / \mathrm{L}(n=24)$ e individuos control sin las condiciones anteriores $(n=20)$. Resultados: los resultados revelaron que existe una correlación positiva y estadísticamente significativa entre los valores de la concentración de TNF- $\alpha$ y albuminuria $(p<0,03)$. Entre los niveles de adiponectina y de TNF- $\alpha$ aparentemente no existe ningún tipo de correlación $(p>0,05)$. Conclusiones: aunque no existen diferencias significativas entre los niveles de adiponectina y de TNF- $\alpha$, el TNF- $\alpha$ podría ser explotado como un marcador temprano de daño renal o servir para el diseño de estimadores de la progresión de la lesión en los pacientes con diabetes mellitus tipo 2.
\end{abstract}

Palabras clave: diabetes mellitus, factor de necrosis tumoral alfa, adiponectina, albuminuria, enfermedades renales.

Sierra-Castrillo J, Gómez-Rave LJ. Asociación de los niveles de adiponectina y del factor de necrosis tumoral-alfa (TNF- $\alpha$ ) con la albuminuria en pacientes con diabetes mellitus tipo 2. Medicina \& Laboratorio 2017; 23: 257-270.

${ }^{1}$ Bacterióloga y Laboratorista Clínica, MSc en Bioquímica Clínica. Docente de Investigación, Grupo Biogen, programa de Bacteriología, Universidad de Santander. Cúcuta, Colombia. Correspondencia: Avenida 4 Calle 10N Urbanización El Bosque. Correo electrónico: jho.sierra@mail.udes.edu.co jhosica1988@hotmail.com

${ }^{2}$ Química Farmacéutica, MSc en Bioquímica Clínica. Investigadora Grupo Biociencias, Facultad de Ciencias de la Salud, Colegio Mayor de Antioquia. Medellin, Colombia.

Conflicto de intereses: los autores declaran que no tienen conflicto de intereses Medicina \& Laboratorio 2017; 23: 257-270

Módulo 19 (Investigación), número 58. Editora Médica Colombiana S.A. 2017 Recibido el 21 de mayo de 2017; aceptado el 29 de junio de 2017 
La diabetes mellitus tipo 2 es una enfermedad crónica caracterizada por hiperglicemia [1-3], ser secundaria a un proceso de insilunorresistencia y deficiencia de insulina [2]. Cuando la hiperglicemia no es controlada puede llevar a una nefropatía diabética, una complicación cuyo mecanismo etiológico aún no es bien conocido, pero que al parecer está estrechamente ligada a trastornos vasculares de la circulación renal y al aumento de citoquinas proinflamatorias que alteran la estructura y función del glomérulo [3,4].

El aumento de glucosa en sangre activa varios sistemas del metabolismo celular donde se produce acumulación excesiva de especies reactivas de oxígeno a través de la aceleración de la cadena respiratoria y el aumento de función de las enzimas xantina oxidasa, NADH/NADPH oxidasa, óxido nítrico sintasa (NOS) y ciertas hemoproteínas [4]. Particularmente, las células mesangiales del glomérulo son las más susceptibles ya que son incapaces de contrarrestar las altas concentraciones intracelulares de glucosa durante la hiperglucemia, lo que las somete a un estrés oxidativo mediado por el aumento anormal de especies reactivas de oxígeno [5].

También se producen alteraciones en la hemodinamia renal que incrementan la presión hidrostática capilar y deterioran la barrera de filtración, compuesta por células endoteliales, una lámina basal y podocitos, lo que en conjunto se traduce en una hiperfiltración glomerular [6]. De esta manera, mayores cantidades de proteínas pasan al espacio de Bowman, entre ellas la albúmina, cuyos niveles en orina se incrementan por encima de los $30 \mathrm{mg} /$ día, valor alrededor del cual oscila el límite superior de la concentración normal y que ha sido útil en el diagnóstico de nefropatías como signo clínico principal [7].
Se define como normoalbuminuria a la excreción urinaria de esta proteína menor a 30 $\mathrm{mg} /$ día, microalbuminuria cuando su valor se encuentra entre $30 \mathrm{mg} /$ día y $299 \mathrm{mg} /$ día, la cual es considerada una de las primeras manifestaciones de la enfermedad, puede estar condicionada por el ejercicio y en personas sanas tiene poco valor predictivo, y macroalbuminuria o proteinuria cuando la excreción urinaria de albúmina es mayor de $300 \mathrm{mg} /$ día, condición bajo la que se observa deterioro progresivo de la función renal y que puede llevar a cuadros nefróticos cuando la excreción es mayor o igual a 3.500 mg/día [8].

De otro modo, existen múltiples factores que predisponen al desarrollo de diabetes tipo 2 , uno de ellos es el incremento en los niveles de metabolitos, como los lípidos, los ácidos grasos y diversas citoquinas provenientes del tejido adiposo [9], los cuales propician el desarrollo de estados inflamatorios, la activación de leucocitos, monocitos y macrófagos, que a su vez se encuentran vinculados con la resistencia a la insulina, y la pérdida de función secretora de esta hormona en los islotes pancreáticos de Langerhans $[9,10]$.

Los macrófagos que residen en el tejido adiposo son de dos tipos: los M1, que se encuentran generalmente en estado activo, secretan citoquinas proinflamatorias, como el factor de necrosis tumoral-alfa (TNF- $\alpha$ ) y las interleuquinas 6 y 8 (IL-6 e IL-8), y pueden producir óxido nítrico sintetasa inducible (iNOS), especies reactivas de oxígeno y resistencia a la insulina; y los $M 2$, que se activan regularmente, producen IL-10 e IL-1 y han sido implicados en la remodelación tisular. El exceso de tejido adiposo, entonces, provoca un desequilibrio en los tipos de macrófagos induciendo la diferenciación de los M2 al tipo M1. Como resultado, las citoquinas proinflamatorias estimuladas por el tejido adiposo, 0 adipocitoquinas, y los radicales libres, se 
producen en mayor cantidad, lo que exacerba la resistencia a la insulina, mientras que se reducen ciertos efectos beneficiosos sobre la regulación metabólica sistémica asociados con la función de los M2 [11,12].

Estas adipocitoquinas y otras citoquinas parecen estar involucradas también en el desarrollo y la progresión de la nefropatía diabética, dado que poseen efectos autocrinos, yuxtacrinos y paracrinos característicos que les permiten coordinar y propagar la respuesta inflamatoria en varios tejidos $[9,10,13]$. En este contexto se le ha atribuido al tejido adiposo funciones endocrinas, ya que algunas de las moléculas que secreta no solo son inmunomoduladoras, sino que pueden regular en cierto grado los lípidos sistémicos, la homeostasis de la glucosa y la relación con otros órganos clave del metabolismo, como el hígado, el páncreas, el músculo y el sistema nervioso central, lo que podría explicar las anomalías metabólicas en otros tejidos cuando hay disfunciones en las adipocitoquinas [13].

Cabe aclarar que la inflamación del tejido adiposo es importante para que el organismo se pueda adaptar al exceso de energía almacenada y así mantener la homeostasis metabólica [14]. Algunas adipocitoquinas secretadas por el tejido adiposo blanco como la resistina y la adiponectina están implicadas en la regulación del peso corporal. Estudios han demostrado que el efecto regulador de la hormona leptina se logra gracias a la emisión de señales transmitidas hacia el hipotálamo, estructura que libera, posteriormente, neuropéptidos y neurotransmisores moduladores de la ingesta de alimentos y el gasto energético [15]. Esta hormona también es conocida por su acción antidiabética, por la regulación que puede ejercer sobre la lipogénesis hepática a través de la supresión de la expresión de enzimas necesarias para la síntesis de ácidos grasos y por inducir el aumento de la oxidación de estos últimos en el músculo [16].

La hormona adiponectina, otra adipocitoquina ampliamente secretada, mejora la sensibilidad a la insulina, exhibe propiedades antiaterogénicas, cardioprotectoras y antinflamatorias. Además, activa las vías de señalización mediante la unión a sus receptores adipo-R1, los cuales actúan como mediadores del efecto sensibilizante a la insulina en el hígado y el músculo, así como de la oxidación de los ácidos grasos, y a los receptores adipo-R2, que aumentan la oxidación de los ácidos grasos en el hígado [17]. La adiponectina facilita, también, la opsonización de las células apoptóticas y su posterior absorción por los macrófagos [18], inhibe la actividad del factor nuclear-k $\beta$ (NF- $\kappa \beta$ ), lo que conduce a la reducción de la adhesión de los monocitos a las células endoteliales $[19,20]$ y, al mismo tiempo, suprime la apoptosis de estas, lo que favorece la cardioprotección [21].

Otros efectos menos estudiados de la adiponectina están relacionados con la acción supresora que ejerce sobre la producción del TNF- $\alpha$, un factor regulador del metabolismo de los adipocitos clave en el proceso de inflamación, que no solo proviene de la expresión en el tejido adiposo, sino que también implica aquel que es derivado de los macrófagos y otras células inmunes. El TNF- $\alpha$ disminuye la lipogénesis, aumenta la lipólisis, induce hiperglucemia y resistencia a la insulina por interferir con su receptor y con la activación de ciertas vías de señalización. Estudios sugieren que la expresión del TNF- $\alpha$ en los macrófagos es inducido por los ácidos grasos libres [22] y que este, posteriormente, estimula la lipólisis, lo que incrementa la liberación de ácidos grasos de los adipocitos y establece, 
de esta manera, una relación cíclica ácidos grasos-adipocitoquina que se autoperpetúa para potenciar los efectos ya mencionados en la inflamación metabólica [23].

Tanto el TNF- $\alpha$ como la adipocitoquina proinflamatoria IL-6 están implicados en el desarrollo de la citotoxicidad renal debido a que inducen lesión celular directa, apoptosis, engrosamiento de la membrana basal glomerular y aumento de la permeabilidad endotelial y de la fibronectina. Los mayores niveles de IL-6 se han evidenciado en las células mesangiales, en el intersticio y en los túbulos renales de pacientes con nefropatía diabética, lo que la posiciona como un marcador predictor de la gravedad y el estadio de la enfermedad. Incluso, se ha sugerido que la IL-6 participa favorablemente en el proceso de resistencia a la insulina y en el mantenimiento del metabolismo de la glucosa en todo el organismo [24].

La adiponectina, posiblemente, no solo actúe como regulador negativo del TNF- $\alpha$, como ya se expuso, sino que también influya del mismo modo sobre la expresión de la IL-6 debido a sus propiedades antinflamatorias. Dada esta relación se planteó si podría ser válido utilizar los niveles de adiponectina y TNF- $\alpha$ en sangre como marcadores de la severidad del daño renal en pacientes con diabetes mellitus tipo 2 . Con base en esta pregunta se realizó un estudio en el Hospital Universitario Erasmo Meoz de la ciudad de Cúcuta (Colombia) con pacientes diabéticos e individuos control a quienes se les determinaron sus concentraciones de adiponectina y TNF- $\alpha$ en sangre, las cuales, posteriormente, se correlacionaron con los niveles de albuminuria y el tiempo desde el diagnóstico de la diabetes para así determinar si las concentraciones de estas citoquinas podían servir como marcadores de daño renal en este tipo de pacientes.

\section{Materiales y métodos}

\section{Población y tipo de estudio}

La investigación se basó en un modelo descriptivo de corte transversal, con una población de estudio conformada por pacientes con diagnóstico de diabetes mellitus tipo 2 y albuminuria mayor que $30 \mathrm{mg} / \mathrm{L}$ que ingresaron al Hospital Universitario Erasmo Meoz (Cúcuta, Colombia) durante el periodo de mayo a octubre del 2015, de acuerdo con los lineamientos requeridos para trabajos de investigación con poblaciones humanas y bajo la aprobación del comité de ética de la ESE Hospital Universitario Erasmo Meoz, radicado N. ${ }^{\circ} 13223$. De forma adicional se incluyó un grupo control conformado por individuos que no padecían diabetes mellitus tipo 2 ni albuminuria, que consultaron en la misma institución por otras causas durante el periodo establecido y que fueron reclutados de forma voluntaria para poder establecer las comparaciones con el grupo de pacientes diabéticos.

La información relativa a la edad, el sexo, el peso, la talla, los antecedentes familiares, la situación laboral, los hábitos alimenticios, el consumo de alcohol, el tabaquismo, la actividad física y el consumo de medicamentos fue obtenida mediante el diligenciamiento de una encuesta que incluía; además, un acta de consentimiento informado.

Los criterios de inclusión utilizados en la selección de los pacientes con diagnóstico de diabetes tipo 2 fueron: personas mayores de 35 años, sin enfermedad cardiaca ni pulmonar, sin ningún tipo de cáncer ni colesterol total alto; además, con niveles de albuminuria mayor a $30 \mathrm{mg} / \mathrm{L}$. Los criterios de inclusión utilizados en la selección de los pacientes controles fueron: personas mayores de 35 años de edad, con composición corporal sin obesidad 
(índice de masa corporal (IMC) menor que 30), sin diagnóstico previo de diabetes ni alteración en su glicemia en ayunas confirmada por prueba de laboratorio, sin daño renal, sin enfermedad cardiaca ni pulmonar, sin ningún tipo de cáncer ni colesterol alto.

\section{Determinación de glicemia}

A los pacientes seleccionados para el estudio se les extrajo sangre en ayunas mediante punción venosa en tubos secos. Las muestras se centrifugaron para obtener el suero en el laboratorio y se determinaron los niveles de glucosa por el método de glucosa-oxidasa en el analizador químico automatizado CB3500 I (Wiener Lab, Rosario, Argentina). El resto del suero de las muestras se depositó en viales marcados y se almacenaron a $-80{ }^{\circ} \mathrm{C}$ hasta un posterior análisis.

\section{Determinación de albuminuria}

Se le solicitó, tanto a los casos como a los controles, la recolección de la primera orina de la mañana por micción espontánea a las que se les determinaron cuantitativamente los niveles de albúmina utilizando un método inmunoturbidimétrico de la casa comercial Wiener Lab (Rosario, Argentina) en el equipo CB3500 I, que permite discriminar entre normoalbuminuria (0 - $30 \mathrm{mg} / \mathrm{día})$, microalbuminuria (30 - $299 \mathrm{mg} /$ día) y macroalbuminuria o proteinuria ( $<300 \mathrm{mg} /$ día).

\section{Determinación de adiponectina y TNF- $\alpha$}

Los sueros guardados en viales a $-80{ }^{\circ} \mathrm{C}$ se descongelaron luego de la recolección de la totalidad de las muestras para determinarles cuantitativamente los niveles de adiponectina y TNF- $\alpha$ mediante ensayos de inmunoabsorción ligados a enzimas (ELISA tipo sándwich), empleando un estuche de la casa comercial eBioscience-Thermo Fisher Scientific (Waltham, Massachusetts, Estados Unidos) y los equipos de lectura Choromate 4300, de lavado STAT FAX 2600 e incubadora STAT FAX 2200 (Awareness Technology, Inc., Palm City, Florida, Estados Unidos).

La ELISA para la determinación de la adiponectina humana tiene un límite de detección de $0,012 \mathrm{ng} / \mathrm{mL}$, un coeficiente de variabilidad intraensayo de $4,2 \%$ e interensayo de 3,1\%. La ELISA para la detección del TNF$\alpha$ tiene un límite de detección de $2,3 \mathrm{pg} / \mathrm{mL}$ y un coeficiente de variabilidad intraensayo de $6,0 \%$ e interensayo de $7,4 \%$.

\section{Análisis estadístico}

El análisis estadístico se basó en la representación de la información de manera gráfica en diagramas de barra, distribuciones de frecuencia simple y tablas de contingencia. Se realizó el cálculo de medidas descriptivas como promedios y desviación estándar, prueba de hipótesis para diferencia de promedios, así como el cálculo de los coeficientes de correlación de Pearson ( $R$ de Pearson) y de Spearman ( $R$ de Spearman) para las variables de interés, bajo un nivel de significancia de 0,05.

\section{Resultados}

\section{Características demográficas de la población de estudio}

La muestra estuvo conformada por 44 sujetos que consultaron durante el periodo establecido. De acuerdo con los criterios de selección, previamente establecidos, los grupos de análisis estuvieron conformados por 24 pacientes con diagnóstico de diabetes mellitus tipo 2 y albuminuria por encima de $30 \mathrm{mg} / \mathrm{L}$ (grupo casos) y 20 individuos 
sin las condiciones anteriores (grupo control). El $41,7 \%$ de los casos eran varones y el $58,3 \%$ eran mujeres, mientras que en el grupo control el $50 \%$ eran mujeres y el $50 \%$ hombres (véase tabla 1).

\begin{tabular}{|c|c|c|c|}
\hline \multirow{3}{*}{ Grupo } & \multicolumn{2}{|c|}{ Género } & \multirow[b]{2}{*}{ Total } \\
\hline & Masculino & Femenino & \\
\hline & N. ${ }^{\circ}(\%)$ & N..$^{\circ}(\%)$ & N. ${ }^{\circ}(\%)$ \\
\hline Diabéticos & $10(41,7)$ & $14(58,3)$ & $24(54,5)$ \\
\hline Controles & $10(50,0)$ & $10(50,0)$ & $20(45,5)$ \\
\hline Total & $20(45,5)$ & $24(54,5)$ & $44(100,0)$ \\
\hline
\end{tabular}

La edad promedio en los diabéticos fue de $62,7 \pm 10,5$ años y en los controles de 63,3 \pm 10,4 años. Respecto al peso corporal el promedio en los diabéticos fue de $70,8 \pm 9,0 \mathrm{~kg}$, mientras que en el grupo control fue de 63,9 $\pm 10,8 \mathrm{~kg}$, con valores que variaron entre 45 $\mathrm{kg}$ y $99 \mathrm{~kg}$. La estatura promedio en el grupo de pacientes diabéticos fue de 1,66 $\pm 0,09$ metros $y$ en el grupo control fue de 1,57 \pm 0,16 metros. El índice de masa corporal promedio en el grupo de casos fue de 26,04 \pm 4,54 ; en el grupo control fue de $27,3 \pm 11,8$.

Como se puede ver en la tabla $\mathbf{2}$ se evidenciaron diferencias significativas entre el grupo control y el grupo de diabéticos en relación con el peso y la estatura $(p<0,05)$; sin embargo, respecto al índice de masa corporal se observó un comportamiento similar entre los dos grupos $(p=0,64)$. Tampoco se notaron diferencias respecto a la edad $(p=0,84)$.

En el grupo de diabéticos la mediana de tiempo del diagnóstico de la enfermedad fue de 144 meses, con un intervalo que oscilaba entre 5 y 40 años. La mayoría (45,8\%) de los pacientes llevaba entre 10 y 15 años con el diagnóstico y el 12,6\% llevaba más de 20 años, mientras que los demás $(41,6 \%)$ llevaban menos de 10 años con este.

\begin{tabular}{|c|c|c|c|c|}
\hline \multirow[t]{2}{*}{ Variable } & \multirow[t]{2}{*}{ Grupo } & \multicolumn{2}{|c|}{$\begin{array}{c}\text { Medidas } \\
\text { descriptivas }\end{array}$} & \multirow[t]{2}{*}{ Valor $p$} \\
\hline & & N. ${ }^{\circ}$ & Promedio & \\
\hline \multirow{2}{*}{$\begin{array}{l}\text { Edad } \\
\text { (años) }\end{array}$} & Diabéticos & 24 & $62,71 \pm 10,51$ & \multirow[t]{2}{*}{0,84} \\
\hline & Controles & 20 & $63,35 \pm 10,43$ & \\
\hline \multirow[t]{2}{*}{ Peso (kg) } & Diabéticos & 24 & $70,79 \pm 9,04$ & \multirow[t]{2}{*}{0,03} \\
\hline & Controles & 20 & $63,90 \pm 10,84$ & \\
\hline \multirow[t]{2}{*}{ Talla (m) } & Diabéticos & 24 & $1,66 \pm 0,09$ & \multirow[t]{2}{*}{0,02} \\
\hline & Controles & 20 & $1,57 \pm 0,16$ & \\
\hline \multirow{2}{*}{$\begin{array}{l}\text { Índice } \\
\text { de masa } \\
\text { corporal }\end{array}$} & Diabéticos & 24 & $26,04 \pm 4,54$ & \multirow[t]{2}{*}{0,64} \\
\hline & Controles & 20 & $27,27 \pm 11,80$ & \\
\hline
\end{tabular}

\section{Niveles de adiponectiva, TNF- $\alpha$ y albuminuria}

Al comparar los niveles de albuminuria, adiponectina y TNF- $\alpha$ entre el grupo de pacientes y el grupo control se logró determinar que el nivel medio de adiponectina presentaba resultados similares en ambos grupos $(p=0,43)$ y que el nivel medio de TNF- $\alpha$ era significativamente mayor en el grupo de pacientes diabéticos respecto al grupo control $(p=0,04)$, con un promedio de 13,79 \pm 20 $\mathrm{pg} / \mathrm{mL}$ frente a $5,2 \pm 2,7 \mathrm{pg} / \mathrm{mL}$. En cuanto a la albuminuria, esta fue mayor en los pacientes diabéticos de forma estadísticamente significativa $(p=0,00)$ (véase tabla 3).

Al analizar las correlaciones existentes entre los valores para TNF- $\alpha$, adiponectina, albuminuria, la edad y el género en los pacientes diabéticos se logró determinar que no se evidenciaba una relación estadísticamente significativa entre la edad o el género y los niveles de albuminuria, adiponectina y 


\begin{tabular}{|c|c|c|c|c|}
\hline \multirow[t]{2}{*}{ Variable } & \multirow[t]{2}{*}{ Grupo } & \multicolumn{2}{|c|}{$\begin{array}{l}\text { Medidas } \\
\text { descriptivas }\end{array}$} & \multirow[t]{2}{*}{ Valor $p$} \\
\hline & & N..$^{\circ}$ & Promedio & \\
\hline \multirow[t]{2}{*}{$\begin{array}{l}\text { Albuminuria } \\
\text { (mg/día) }\end{array}$} & Diabéticos & 24 & $\begin{array}{l}60,02 \pm \\
16,68\end{array}$ & 0,00 \\
\hline & Controles & 20 & $\begin{array}{l}8,01 \pm \\
7,45\end{array}$ & \\
\hline \multirow[t]{2}{*}{$\begin{array}{l}\text { Adiponectina } \\
\text { (ng/mL) }\end{array}$} & Diabéticos & 24 & $\begin{array}{l}4,22 \pm \\
2,04\end{array}$ & 0,43 \\
\hline & Controles & 20 & $\begin{array}{l}4,68 \pm \\
1,76\end{array}$ & \\
\hline \multirow[t]{2}{*}{$\begin{array}{l}\text { TNF- } \alpha \\
(\mathrm{pg} / \mathrm{mL})\end{array}$} & Diabéticos & 24 & $\begin{array}{l}13,79 \pm \\
20,04\end{array}$ & 0,04 \\
\hline & Controles & 20 & $\begin{array}{l}5,21 \pm \\
2,73\end{array}$ & \\
\hline
\end{tabular}

TNF- $\alpha(p>0,05)$, pero que existe una correlación positiva significativa entre los valores para la concentración de adiponectina y de albuminuria en los pacientes diabéticos ( $p$ =0,03); es decir, a mayor concentración de adiponectina mayor nivel de albuminuria. De igual manera, existe una correlación positiva significativa entre los valores para la concentración de TNF- $\alpha$ y de albuminuria ( $p$ $<0,03$ ), lo que indica que a mayor nivel de concentración de TNF- $\alpha$ mayor nivel de albuminuria. No se evidenció correlación significativa entre la concentración de TNF- $\alpha$ y la concentración de adiponectina en estos pacientes $(p=0,18)$ (véase tabla 4$)$.

\section{Discusión}

La diabetes mellitus tipo 2 se asocia con complicaciones de origen cardiovascular, renal, ocular y neurológico $[25,26]$. Entre las renales se encuentra la nefropatía diabética, caracterizada por el aumento en la presión de la filtración, la tasa de excreción de la albúmina en la orina y la disminución de la función renal. Los niveles elevados de albúmina, por lo tanto, sirven como un indicador de la progresión del daño renal [27].

Mediante un estudio transversal, que incluyó sujetos con diabetes mellitus tipo 2 y albuminuria, y sujetos sin ninguna de estas dos condiciones, se buscó dilucidar el tipo de relación existente entre los niveles de albúmina en orina y dos proteínas en suero, la adiponectina y el TNF- $\alpha$. La adiponectina es una hormona que participa en la regulación del metabolismo de la glucosa y los ácidos grasos, y en la adquisición de una mayor sensibilidad a la insulina. El TNF- $\alpha$ está implicado en los procesos de resistencia a la insulina debido a que causa interferencia en la ruta de señalización que se activa tras la unión de la insulina a su receptor $[28,29]$.

En los resultados encontrados la albuminuria promedio presentada en los pacientes con diabetes tipo 2 fue de 60,02 mg/L, valor que fue estadísticamente significativo y predecible, respecto a los controles, pues este parámetro se tuvo en cuenta solo como criterio de selección de la población. Este hallazgo corrobora el efecto que puede tener la enfermedad diabética sobre el sistema renal. Algunos autores señalan que la presencia de albuminuria en la diabetes abarca otras alteraciones estructurales caracterizadas por hipertrofia temprana del glomérulo y los túbulos renales, el engrosamiento de la membrana basal glomerular y de la membrana tubular y la acumulación de componentes de la matriz extracelular en el mesangio [30]. Por tal razón, en los pacientes con diabetes tipo 2 y que presentan albuminuria se podría evaluar el grado de nefropatía a partir de la valoración de la tasa de filtración glomerular estimada (EGFR). Esta información cuantitativa mejora la predicción de la enfermedad renal terminal en 


\begin{tabular}{|c|c|c|c|c|c|c|c|}
\hline \multirow[t]{2}{*}{ Grupo } & \multirow{2}{*}{\multicolumn{2}{|c|}{ Variables }} & \multicolumn{3}{|l|}{ Casos } & \multirow[b]{2}{*}{$\begin{array}{l}\text { Concentración } \\
\text { adiponectina } \\
(\mathrm{ng} / \mathrm{mL} \text { ) }\end{array}$} & \multirow[b]{2}{*}{$\begin{array}{l}\text { Concentración } \\
\text { TNF- } \alpha(\mathrm{pg} / \mathrm{mL})\end{array}$} \\
\hline & & & Edad & Género & $\begin{array}{l}\text { Albuminuria } \\
\text { (mg/L) }\end{array}$ & & \\
\hline \multirow[t]{15}{*}{ Casos } & \multirow[t]{3}{*}{ Edad } & $\begin{array}{l}\text { Coeficiente de } \\
\text { correlación }\end{array}$ & N.A. & $-0,02$ & 0,07 & 0,13 & $-0,25$ \\
\hline & & Significancia & N.A. & 0,46 & 0,37 & 0,27 & 0,12 \\
\hline & & $\mathrm{N}$ & N.A. & 24 & 24 & 24 & 24 \\
\hline & \multirow[t]{3}{*}{ Género } & $\begin{array}{l}\text { Coeficiente de } \\
\text { correlación }\end{array}$ & 0,04 & N.A. & $-0,06$ & 0,05 & 0,28 \\
\hline & & Significancia & 0,42 & N.A. & 0,39 & 0,41 & 0,09 \\
\hline & & $\mathrm{N}$ & 24 & N.A. & 24 & 24 & 24 \\
\hline & \multirow[t]{3}{*}{$\begin{array}{l}\text { Albuminuria } \\
\text { (mg/L) }\end{array}$} & $\begin{array}{l}\text { Coeficiente de } \\
\text { correlación }\end{array}$ & 0,07 & $-0,01$ & N.A. & 0,39 & 0,39 \\
\hline & & Significancia & 0,37 & 0,48 & N.A. & 0,03 & 0,03 \\
\hline & & $\mathrm{N}$ & 24 & 24 & N.A. & 24 & 24 \\
\hline & \multirow{3}{*}{$\begin{array}{l}\text { Concentra- } \\
\text { ción adipo- } \\
\text { nectina (ng/ } \\
\mathrm{mL} \text { ) }\end{array}$} & $\begin{array}{l}\text { Coeficiente de } \\
\text { correlación }\end{array}$ & 0,13 & 0,02 & 0,39 & N.A. & 0,19 \\
\hline & & Significancia & 0,27 & 0,46 & 0,03 & N.A. & 0,18 \\
\hline & & $\mathrm{N}$ & 24 & 24 & 24 & N.A. & 24 \\
\hline & \multirow{3}{*}{$\begin{array}{l}\text { Concentra- } \\
\text { ción TNF- } \alpha \\
(\mathrm{pg} / \mathrm{mL})\end{array}$} & $\begin{array}{l}\text { Coeficiente de } \\
\text { correlación }\end{array}$ & $-0,25$ & 0,28 & 0,39 & 0,19 & N.A. \\
\hline & & Significancia & 0,12 & 0,09 & 0,03 & 0,18 & N.A. \\
\hline & & $\mathrm{n}$ & 24 & 24 & 24 & 24 & N.A. \\
\hline \multicolumn{8}{|c|}{ N.A. $=$ No Aplica } \\
\hline
\end{tabular}

poblaciones en las que la diabetes mellitus tipo 2 representa el factor etiológico principal [31].

Ahora bien, es importante tener en cuenta que al parecer existen diferentes fenotipos expresados de manera frecuente en los pacientes diabéticos que pueden conducir a nefropatía diabética sin pérdida de proteínas. Por lo tanto, no siempre la microalbuminuria y la macroalbuminuria aparecerán como consecuencia del deterioro de la función renal. Se ha determinado que durante la enfermedad renal en pacientes con diabetes mellitus tipo 2 hay alto grado de heterogenicidad histológica, lo que permitiría explicar la ausencia de la relación con la pérdida de proteínas en orina. De esta manera, se recomienda no excluir a los pacientes con diabetes mellitus tipo 2 sin albuminuria de los estudios que pretendan valorar la enfermedad renal; además, es importante realizar más estudios sobre este fenotipo y el papel que desempeña [32].

Si bien el promedio de edad de los controles fue mayor que el de los pacientes diabéticos, la diferencia no fue significativa $(p>0,05)$. En cuanto al peso y la talla aunque se observaron diferencias significativas en el análisis de medias de los grupos $(p<0,05)$, donde los casos mostraron un valor mayor en ambas 
variables, ., el índice de masa corporal es el que proporciona la medida más útil para la determinación de la condición de sobrepeso y obesidad en la población, y que en el presente estudio no presentó diferencias significativas entre los grupos de estudio $(p>0,05)$. Sin embargo, se debe considerar en estudios futuros evaluar aspectos como la localización de la grasa corporal y la medida del perímetro abdominal en los pacientes diabéticos en comparación del grupo control, ya que la obesidad central está más correlacionada con las complicaciones metabólicas e inflamatorias [33]. Yi-Jing Sheen y colaboradores (2014) [34] concluyeron que entre los factores de riesgo asociados a la diabetes tipo 2, que llevan a la disminución de la función renal, se encuentran la edad, el peso, la etnia, la genética, el estilo de vida, el aumento en la glucemia, las dislipidemias, la albuminuria avanzada y otras alteraciones bioquímicas del individuo.

Por su parte, Nogueira y colaboradores (2015) [35], en un estudio transversal realizado en 1.320 pacientes con diabetes mellitus, determinaron que el tiempo de diagnóstico presenta una asociación directa con el desarrollo de las complicaciones relacionadas con la enfermedad tales como amputación, pie diabético, nefropatía, retinopatía diabética, infarto agudo de miocardio y accidentes cerebrovasculares. Estas complicaciones se presentan con mayor frecuencia en los pacientes con tiempo de diagnóstico superior a 10 años. En el presente estudio se pudo observar que la mayoría de los pacientes diabéticos tenían un tiempo de diagnóstico de 10 a 15 años, lo que contribuyó a predecir un posible daño renal como proceso secundario a la diabetes.

Al comparar la concentración de adiponectina en los grupos se evidenció que es mayor en el grupo control $(4,68 \mathrm{ng} / \mathrm{mL}$ frente 4,22 $\mathrm{ng} / \mathrm{mL}$ ), pero sin significancia estadística ( $p$ $=0,43$ ), lo que indica que probablemente las dos poblaciones no guardan grandes discrepancias respecto a este parámetro. Al hacer el análisis bivariado entre las concentraciones de esta hormona y los niveles de albuminuria en los pacientes diabéticos se pudo observar una correlación directa entre ambos analitos $(p=0,03)$. Esto confronta los hallazgos de Yano y colaboradores (2007) [36], donde el resultado de la asociación entre la concentración de adiponectina y el nivel de albuminuria en pacientes delgados y con obesidad mostró que la albúmina en orina se correlacionaba inversamente con la concentración de adiponectina en los individuos obesos.

Probablemente, estas diferencias estén ligadas a las características poblacionales, en las que criterios como la obesidad desempeñan un papel clave en la disminución de la adiponectina. Cabe aclarar que en este estudio solo el $16,7 \%$ de los pacientes diabéticos fueron catalogados como obesos. Es importante destacar también que si en un paciente obeso disminuyen los niveles de adiponectina sérica basal puede tener mayor predisposición al desarrollo de diabetes mellitus tipo 2 [26].

En otro estudio se evidenció que los niveles séricos de adiponectina fueron significativamente menores en los sujetos no diabéticos obesos en comparación con los sujetos diabéticos tipo 2 no obesos $(p=0,006)$ y los sujetos no obesos no diabéticos $(p=0,002)$, sin diferencia significativa al comparar estos dos últimos grupos entre sí $(p>0,05)$, lo que llevó a pensar que las alteraciones de la adiponectina en suero pueden ser debidas al exceso de tejido adiposo [27]. Un hallazgo reciente sugiere que los niveles de 
adiponectina en pacientes diabéticos tipo 2 parecen estar más asociados a la obesidad y menos a la diabetes [28].

Similares a los hallazgos del presente estudio están los de Kato y colaboradores (2008) [37], quienes reportaron que los niveles de adiponectina sérica eran mayores en los sujetos con macroalbuminuria o insuficiencia renal crónica que en aquellos con normoalbuminuria y microalbuminuria. De igual manera, Von Eynatten y colaboradores (2009) [38] evidenciaron la elevación de los niveles de adiponectina en pacientes con enfermedad renal en etapa terminal o síndrome nefrótico. Una posible razón para estos hallazgos puede ser que el aclaramiento renal, que al parecer contribuye con el exceso de esta proteína en la circulación, también se relaciona con el mecanismo de acción de la adiponectina en el riñón, donde aumenta la actividad de la proteína quinasa activada por AMP (AMPK) y la NADPH oxidasa [39]. No obstante, se necesitan varios estudios adicionales para comprender mejor esta vía de señalización y las proteínas diana de la adiponectina. Hasta la fecha, el papel exacto del riñón en la biodegradación y excreción de la adiponectina aún no está claro [40].

De acuerdo con lo anterior, es permisible decir que la asociación de la adiponectina con la función renal en individuos con diabetes tipo 2 aún no se encuentra bien descrita, y en la literatura disponible se han centrado principalmente en el estudio de pacientes con intolerancia a la glucosa o enfermedad renal en etapa terminal. Aunque no es posible aceptar o rechazar la hipótesis de que la adiponectina puede servir como marcador temprano de daño renal en los pacientes diabéticos tipo 2 , sí se puede pensar que su correlación directa con los niveles de albuminuria quizá provenga de la inducción de un mecanismo nefroprotector en el organismo.

Por otra parte, la presente investigación no mostró correlaciones significativas entre la edad y el género con los valores para la adiponectina y la albuminuria $(p>0,05)$ en estos grupos, pero vale la pena decir que en varios estudios se ha evidenciado cómo los niveles de adiponectina se correlacionan positivamente con la edad [41,42]. Según Aleidi y colaboradores (2014) [41] esto se podría explicar debido a que el aumento de la adiponectina, con la edad, se produce en función de la disminución de las hormonas sexuales esteroideas. A esto se suma el aumento del tejido adiposo, que como bien se ha dicho, es un órgano endocrino clave en la expresión de esta adipocitoquina. En el grupo control no se encontraron correlaciones entre las concentraciones de albúmina en orina y las de adiponectina en suero $(p>0,05)$.

Respecto al TNF- $\alpha$ los niveles fueron mayores en los pacientes diabéticos al compararse con el grupo control, obteniéndose una diferencia estadística significativa $(p=0,04)$. Sin embargo, la desviación estándar en el grupo de diabéticos arrojó un valor superior a la media, por lo que deben considerarse posibles sesgos generados por la presencia de valores extremos. Algunos datos recientes han puesto de manifiesto que la concentración plasmática de este mediador inflamatorio, y de otros como la IL-6, se aumenta en los estados de diabetes tipo 2 y obesidad, lo que hace crecer los cuestionamientos sobre los mecanismos que subyacen el proceso inflamatorio en estas dos condiciones $[43,44]$. Se ha tratado de esclarecer la expresión y secreción del TNF- $\alpha$ por el tejido adiposo que ha permitido establecer cierta correlación entre el grado de adiposidad y la 
resistencia a la insulina [43]. El primer estudio que relacionó la obesidad con el TNF- $\alpha$ fue publicado por Hotamisligil y colaboradores (1995) [45], quienes dieron paso al inicio de las investigaciones del TNF- $\alpha$ y la inflamación en la obesidad.

Además del incremento en la expresión del TNF- $\alpha$ en el tejido adiposo de sujetos obesos se ha descrito que sus niveles séricos también son elevados [46] y que la pérdida de peso no solo reduce estos niveles, sino que también disminuye la expresión del ARN mensajero del TNF- $\alpha$ en el tejido adiposo [13]. El TNF- $\alpha$ también se sintetiza $y$ se secreta por el músculo, los linfocitos y los macrófagos [37]. Nieto y colaboradores (2008) [47] han demostrado que el TNF- $\alpha$ tiene mayor expresión en los músculos de sujetos con resistencia a la insulina y diabetes mellitus tipo 2 .

En el análisis de datos del presente trabajo se evidenció que existe una correlación positiva y estadísticamente significativa entre los valores de TNF- $\alpha$ y la albuminuria $(p<$ 0,03 ). Este hallazgo guarda relación con algunas de las actividades biológicas demostradas para el TNF- $\alpha$, como las señaladas por Navarro-González y colaboradores (2011) [48], donde al parecer hay una asociación directa e independiente entre los niveles de la citoquina y los marcadores clínicos de daño renal, con aumento de la misma en suero y orina a medida que la nefropatía progresa.

El TNF- $\alpha$ es producido por las células nativas renales activadas (mesangial glomerular, células epiteliales y endoteliales, y las células tubulares) y por los monocitos/macrófagos activados [10] para aumentar la liberación de otras citoquinas, quimioquinas, factores de crecimiento y proteínas de fase aguda
[49]. Omote y colaboradores (2014) [50] describen al TNF- $\alpha$ como una citoquina pleiotrópica que ejerce múltiples efectos y puede contribuir al desarrollo de la nefropatía diabética a través de varios mecanismos, incluida la reducción del flujo sanguíneo glomerular y la tasa de filtración glomerular, la vasoconstricción mediada por la interacción con las uniones intercelulares y la inducción de proteinuria [49,51]. El aumento de la producción del TNF- $\alpha$ también puede producir estrés oxidativo mediante la activación de la nicotinamida adenina dinucleótido fosfato reducido (NADPH) en las células mesangiales [10]. Finalmente, el TNF- $\alpha$ parece tener un efecto apoptótico y citotóxico directo sobre las células glomerulares [51].

Otros investigadores, como Lampropoulou y colaboradores (2014) [52] encontraron niveles elevados de TNF- $\alpha$ en suero tanto en pacientes macroalbuminúricos como en microalbuminúricos en comparación con los normoalbuminúricos. Por su parte, Chen y colaboradores (2017) [53], con base en los resultados de un metanálisis en seis estudios clínicos de casos y controles publicados entre 1999 y 2016, concluyen que las concentraciones séricas de TNF- $\alpha$ aumentan de forma marcada en los pacientes con diabetes mellitus tipo 2 y nefropatía diabética tipo 2, siendo más altas en estos últimos, lo que sugiere una elevada carga inflamatoria en estos pacientes; además, proponen que esta citoquina podría desempeñar un papel significativo en el estudio de la nefropatía diabética, a pesar de que se necesitan más investigaciones al respecto. En el presente trabajo se pudo observar que entre los niveles de adiponectina y de TNF- $\alpha$, aparentemente, no existe ningún tipo de correlación $(p>0,05)$. Finalmente, los resultados obtenidos concuerdan con los de otros estudios citados en relación con los efectos que puede 
producir el aumento de algunas adipocitoquinas en los pacientes diabéticos.

De acuerdo con el objetivo planteado y los resultados obtenidos se pudo concluir que aunque no existen diferencias significativas entre la adiponectina y el TNF- $\alpha$, los niveles de esta última citoquina podrían ser explotados como marcadores tempranos de daño renal o servir para el diseño de estimadores de la progresión del daño en los pacientes con diabetes tipo 2. Las discrepancias entre este estudio y otros reportados en la literatura podrían deberse a circunstancias propias de la investigación, tales como variaciones en las características de la población control y de diabéticos, la severidad de la diabetes tipo 2, el nivel de daño renal y el número muestra, entre otras.

\section{Conclusiones}

La nefropatía secundaria a la diabetes mellitus tipo 2 es una complicación común que aparece como resultado del daño vascular y metabólico de la barrera de filtración glomerular, que puede ser diagnosticada mediante la determinación de la concentración de albúmina en orina, dado que su incremento patológico es una de las principales características de la enfermedad [3,7]. Esta situación se pudo comprobar en los pacientes diabéticos estudiados, donde sus niveles de albúmina en orina fueron superiores y estadísticamente significativos respecto a los controles; además, guardaron una relación positiva y significativa con los niveles de TNF- $\alpha$.

Por otra parte, es comprensible que esta complicación se exacerbe con la obesidad si se concibe, entre otros factores, el tejido adiposo como un órgano endocrino que secreta una amplia gama de adipocitoquinas, principalmente proinflamatorias como el TNF- $\alpha$, el cual está implicado en la resistencia a la insulina, el estrés celular y la apoptosis, y otras sustancias que ejercen cierta regulación negativa sobre la inflamación, como la adiponectina, o que influyen en la homeostasis del almacenamiento de energía, como la leptina y la resistina $[13,15]$. No hubo diferencias significativas entre los niveles de adiponectina de los pacientes diabéticos y los de los controles, hecho que posiblemente se deba a que las fluctuaciones en esta citoquina se ven más influenciadas por la obesidad que por la diabetes [28].

Los niveles de adiponectina parecen elevarse progresivamente conforme se da el daño renal, el cual se evidencia por el aumento de la albúmina en la orina $[37,38]$. Además, se podría deber, entre otros factores, a la inducción de mecanismos nefroprotectores que se estimulan en el organismo durante tales eventos [39].

Aunque en esta investigación se encontraron ciertas correlaciones relevantes, como la presentada entre las concentraciones de TNF- $\alpha$ y los niveles de albuminuria en pacientes diabéticos, que podrían explotarse como estimadores de la progresión del daño renal, se sugiere realizar estudios prospectivos futuros con mayor número de muestra y con criterios de selección más restringidos para establecer si hay relación entre las concentraciones plasmáticas de otras adipocitoquinas, como la adiponectina, y la reducción o el desarrollo de las complicaciones renales en pacientes con diabetes tipo 2.

\section{Agradecimientos}

A la Universidad de Santander (UDES) por patrocinar los recursos financieros a través de la convocatoria interna focalizada. Al Hospital Universitario Erasmo Meoz por facilitar la población y el uso del laboratorio clínico. 


\section{Bibliografía}

1. Goncalves Reis CE, Dullius J. Glycemic acute changes in type 2 diabetics caused by low and high glycemic index diets. Nutr Hosp 2011; 26: 546-552.

2. Khardori R. Type 2 Diabetes Mellitus Clinical Presentation. 2017. Medscape. Disponible: http://emedicine.medscape. com/article/117853-clinical. Consultado: jun 2017

3. Torres-Viloria A, Zacarías-Castillo R. Nefropatía diabética. Rev Hosp Gral Dr M Gea González 2002; 5: 24-32.

4. Kashihara N, Haruna Y, Kondeti VK, Kanwar YS. Oxidative stress in diabetic nephropathy. Curr Med Chem 2010; 17: 4256-4269.

5. Riemer J, Bulleid N, Herrmann JM. Disulfide formation in the ER and mitochondria: two solutions to a common process. Science 2009; 324: 1284-1287.

6. Battilana CA. El riñón en la hipertensión arterial esencial. Rev Perú Cardiol 1997; XXIII: 26-31.

7. Glassock RJ. Is the presence of microalbuminuria a relevant marker of kidney disease? Curr Hypertens Rep 2010; 12: $364-368$.

8. Tagle R, González F, Acevedo M. Microalbuminuria y excreción urinaria de albúmina en la práctica clínica. Rev Méd Chile 2012; 140: 797-805.

9. King GL. The role of inflammatory cytokines in diabetes and its complications. J Periodontol 2008; 79: 1527-1534

10. Navarro-González JF, Mora-Fernandez $\mathbf{C}$. The role of inflammatory cytokines in diabetic nephropathy. J Am Soc Nephrol 2008; 19: 433-442.

11. Gordon S. Alternative activation of macrophages. Nat Rev Immunol 2003; 3: 23-35.

12. Lumeng CN, Bodzin JL, Saltiel AR. Obesity induces a phenotypic switch in adipose tissue macrophage polarization. J Clin Invest 2007; 117: 175-184.

13. Cao H. Adipocytokines in obesity and metabolic disease. J Endocrinol 2014; 220: T47-59.

14. Ye J, McGuinness OP. Inflammation during obesity is not all bad: evidence from animal and human studies. Am J Physiol Endocrinol Metab 2013; 304: E466-477.

15. Zhang X, Zhang G, Zhang H, Karin M, Bai H, Cai D. Hypothalamic IKKbeta/NF-kappaB and ER stress link overnutrition to energy imbalance and obesity. Cell 2008; 135: 61-73.

16. Sharma A, Bartell SM, Baile CA, Chen B, Podolsky RH, Mclndoe RA, et al. Hepatic gene expression profiling reveals key pathways involved in leptin-mediated weight loss in ob/ob mice. PLoS One 2010; 5: e12147.

17. Yamauchi T, Nio Y, Maki T, Kobayashi M, Takazawa T, Iwabu M, et al. Targeted disruption of AdipoR1 and AdipoR2 causes abrogation of adiponectin binding and metabolic actions. Nat Med 2007; 13: 332-339.

18. Takemura Y, Ouchi N, Shibata R, Aprahamian T, Kirbe MT, Summer RS, et al. Adiponectin modulates inflammatory reactions via calreticulin receptor-dependent clearance of early apoptotic bodies. J Clin Invest 2007; 117 375-386.

19. Okamoto Y, Kihara S, Ouchi N, Nishida M, Arita Y, Kumada $\mathbf{M}$, et al. Adiponectin reduces atherosclerosis in apolipoprotein E-deficient mice. Circulation 2002; 106: 2767-2770.
20. Ouchi N, Kihara S, Arita Y, Maeda K, Kuriyama H, Okamoto $Y$, et al. Novel modulator for endothelial adhesion molecules: adipocyte-derived plasma protein adiponectin. Circulation 1999; 100: 2473-2476

21. Kobayashi $\mathbf{H}$, Ouchi $\mathbf{N}$, Kihara $S$, Walsh $\mathrm{K}$, Kumada $\mathbf{M}$, Abe $\mathrm{Y}$, et al. Selective suppression of endothelial cell apoptosis by the high molecular weight form of adiponectin. Circ Res 2004; 94: e27-31.

22. Nguyen MT, Satoh H, Favelyukis $S$, Babendure JL, Imamura T, Sbodio JI, et al. JNK and tumor necrosis factor-alpha mediate free fatty acid-induced insulin resistance in 3T3-L1 adipocytes. J Biol Chem 2005; 280: 35361-35371.

23. Wang S, Soni KG, Semache $\mathbf{M}$, Casavant S, Fortier $\mathbf{M}$, Pan L, et al. Lipolysis and the integrated physiology of lipid energy metabolism. Mol Genet Metab 2008; 95: 117-126.

24. Kim JH, Bachmann RA, Chen J. Interleukin-6 and insulin resistance. Vitam Horm 2009; 80: 613-633.

25. Gaede $\mathbf{P}$, Lund-Andersen $\mathrm{H}$, Parving $\mathrm{HH}$, Pedersen $\mathrm{O}$. Effect of a multifactorial intervention on mortality in type 2 diabetes. N Engl J Med 2008; 358: 580-591.

26. Lin J, Hu FB, Curhan G. Serum adiponectin and renal dysfunction in men with type 2 diabetes. Diabetes Care 2007; 30: 239-244.

27. Parchwani DN, Upadhyah AA. Diabetic Nephropathy: Progression and Pathophysiology. Int J Med Sci Public Health 2012; 1: 59-70.

28. Fernandez-Veledo S, Vila-Bedmar R, Nieto-Vazquez I Lorenzo $\mathbf{M}$. c-Jun $\mathrm{N}$-terminal kinase $1 / 2$ activation by tumor necrosis factor-alpha induces insulin resistance in human visceral but not subcutaneous adipocytes: reversal by liver $\mathrm{X}$ receptor agonists. J Clin Endocrinol Metab 2009; 94: 3583-3593.

29. Gupta D, Varma S, Khandelwal RL. Long-term effects of tumor necrosis factor-alpha treatment on insulin signaling pathway in HepG2 cells and HepG2 cells overexpressing constitutively active Akt/PKB. J Cell Biochem 2007; 100: 593-607

30. Weil EJ, Lemley KV, Mason CC, Yee B, Jones LI, Blouch K, et al. Podocyte detachment and reduced glomerular capillary endothelial fenestration promote kidney disease in type 2 diabetic nephropathy. Kidney Int 2012; 82: 10101017.

31. Berhane AM, Weil EJ, Knowler WC, Nelson RG, Hanson RL. Albuminuria and estimated glomerular filtration rate as predictors of diabetic end-stage renal disease and death. Clin J Am Soc Nephrol 2011; 6: 2444-2451.

32. Porrini E, Ruggenenti $P$, Mogensen CE, Barlovic DP, Praga $\mathbf{M}$, Cruzado JM, et al. Non-proteinuric pathways in loss of renal function in patients with type 2 diabetes. Lancet Diabetes Endocrinol 2015; 3: 382-391.

33. U.S. Department of Health and Human Services, Public Health Service, National Institutes of Health, National Heart Lung and Blood Institute, National Cholesterol Education Program. ATP III Guidelines At-A-Glance Quick Desk Reference. 2001. Disponible: https://www.nhlbi. nih.gov/files/docs/guidelines/atglance.pdf. Consultado: jun 2017.

34. Sheen YJ, Sheu WH. Risks of rapid decline renal function in patients with type 2 diabetes. World J Diabetes 2014; 5: $835-846$ 
35. Nogueira Cortez D, Afonso Reis I, Silva Souza DA, Lopes Macedo MM, de Carvalho Torres H. Complications and the time of diagnosis of diabetes mellitus in primary care. Acta Paul Enferm 2015; 28: 250-255.

36. Yano Y, Hoshide S, Ishikawa J, Hashimoto T, Eguchi K, Shimada K, et al. Differential impacts of adiponectin on low-grade albuminuria between obese and nonobese persons without diabetes. J Clin Hypertens (Greenwich) 2007; 9: 775-782.

37. Kato K, Osawa H, Ochi M, Kusunoki Y, Ebisui O, Ohno K, et al. Serum total and high molecular weight adiponectin levels are correlated with the severity of diabetic retinopathy and nephropathy. Clin Endocrinol (Oxf) 2008; 68: 442-449.

38. von Eynatten M, Liu D, Hock C, Oikonomou D, Baumann M, Allolio B, et al. Urinary adiponectin excretion: a novel marker for vascular damage in type 2 diabetes. Diabetes 2009; 58: 2093-2099.

39. Christou GA, Kiortsis DN. The role of adiponectin in renal physiology and development of albuminuria. J Endocrinol 2014; 221: R49-61.

40. Sweiss N, Sharma K. Adiponectin effects on the kidney. Best Pract Res Clin Endocrinol Metab 2014; 28: 71-79.

41. Aleidi S, Issa A, Bustanji H, Khalil M, Bustanji Y. Adiponectin serum levels correlate with insulin resistance in type 2 diabetic patients. Saudi Pharm J 2015; 23: 250256.

42. Adamczak M, Rzepka E, Chudek J, Wiecek A. Ageing and plasma adiponectin concentration in apparently healthy males and females. Clin Endocrinol (Oxf) 2005; 62: 114118.

43. Tzanavari T, Giannogonas $\mathbf{P}$, Karalis KP. TNF-alpha and obesity. Curr Dir Autoimmun 2010; 11: 145-156.

44. Dandona P, Aljada A, Bandyopadhyay A. Inflammation: the link between insulin resistance, obesity and diabetes. Trends Immunol 2004; 25: 4-7.

45. Hotamisligil GS, Arner P, Caro JF, Atkinson RL, Spiegel- man BM. Increased adipose tissue expression of tumor necrosis factor-alpha in human obesity and insulin resistance. The Journal of Clinical Investigation 1995; 95: 2409-2415.

46. Vinay DS, Kwon BS. The tumour necrosis factor/TNF receptor superfamily: therapeutic targets in autoimmune diseases. Clin Exp Immunol 2011; 164: 145-157.

47. Nieto-Vazquez I, Fernandez-Veledo S, Kramer DK, Vila-Bedmar R, Garcia-Guerra L, Lorenzo M. Insulin resistance associated to obesity: the link TNF-alpha. Arch Physiol Biochem 2008; 114: 183-194.

48. Navarro-González JF, Mora-Fernández C, Muros de Fuentes M, Garcia-Perez J. Inflammatory molecules and pathways in the pathogenesis of diabetic nephropathy. Nat Rev Nephrol 2011; 7: 327-340.

49. Navarro JF, Mora C, Gomez M, Muros M, Lopez-Aguilar C, Garcia J. Influence of renal involvement on peripheral blood mononuclear cell expression behaviour of tumour necrosis factor-alpha and interleukin- 6 in type 2 diabetic patients. Nephrol Dial Transplant 2008; 23: 919-926.

50. Omote K, Gohda T, Murakoshi M, Sasaki Y, Kazuno S, Fujimura T, et al. Role of the TNF pathway in the progression of diabetic nephropathy in KK-A(y) mice. Am J Physiol Renal Physiol 2014; 306: F1335-1347.

51. Wang $\mathbf{Z}$, Wei $\mathbf{M}$, Wang $\mathbf{M}$, Chen $\mathbf{L}$, Liu $\mathbf{H}$, Ren $\mathrm{Y}$, et al. Inhibition of macrophage migration inhibitory factor reduces diabetic nephropathy in type II diabetes mice. Inflammation 2014; 37: 2020-2029.

52. Lampropoulou IT, Stangou M, Papagianni A, Didangelos T, Iliadis F, Efstratiadis G. TNF-alpha and microalbuminuria in patients with type 2 diabetes mellitus. J Diabetes Res 2014; 2014: 394206.

53. Chen YL, Qiao YC, Xu Y, Ling W, Pan YH, Huang YC, et al. Serum TNF-alpha concentrations in type 2 diabetes mellitus patients and diabetic nephropathy patients: A systematic review and meta-analysis. Immunol Lett 2017; 186: 52-58.

\begin{abstract}
Type 2 Diabetes mellitus is a metabolic chronic disease, which if it is not properly controlled produces a progressive kidney damage causing diabetic nephropathy. It is also highly associated with a chronic inflammatory state, related to adipocytokines (IL-6, TNF- $\alpha$, leptin, adiponectin) secretion which are involved in metabolic homeostasis and crucial in both regulating the atherogenic process as in insulin resistance. Objective: To relate the behavior of adiponectin and TNF- $\alpha$ levels with albuminuria in patients with type 2 diabetes mellitus. Materials and methods: The research was based on a descriptive cross-sectional model with a study population composed by patients admitted to University Hospital Erasmo Meoz (Cucuta, Colombia). According to previously established selection criteria, two analysis groups were conformed; patients diagnosed with type 2 diabetes mellitus and albuminuria $>30 \mathrm{mg} / \mathrm{L}(n=24)$, and a control group without the above conditions $(n=20)$. Results: The results revealed that there is a positive and statistically significant correlation between TNF- $\alpha$ concentration and albuminuria ( $p$ <0.03). It was observed that between TNF- $\alpha$ and adiponectin levels there are no apparently correlation ( $p>0.05$ ). Conclusions: although there are no significant differences between adiponectin and TNF- $\alpha$ levels, TNF- $\alpha$ could be exploited as an early markers for kidney damage or help designing of progression estimators of injury in patients with diabetes type 2.
\end{abstract}

Keywords: Diabetes mellitus, tumor necrosis factor-alpha, adiponectin, albuminuria, kidney diseases. 